\title{
Periodized combined training in physically active overweight women over 50 years
}

\author{
Thaís Mara Alexandre Bertazone ${ }^{1}$ (D), Leonardo Henrique de Lima Medeiros ${ }^{2}$ (D), \\ Célia Ignácio de Oliveira $^{3}$ (D), Carlos Roberto Bueno Junior $^{2}$ (D), Angelita Maria Stabile ${ }^{1}$ \\ ${ }^{1}$ Universidade de São Paulo, Escola de Enfermagem de Ribeirão Preto, Ribeirão Preto, SP, \\ Brazil; ${ }^{2}$ Universidade de São Paulo, Escola de Educação Física e Esporte de Ribeirão Preto, \\ Ribeirão Preto, SP, Brazil; ${ }^{3}$ Universidade de São Paulo, Faculdade de Medicina de Ribeirão \\ Preto, Hospital das Clínicas, Ribeirão Preto, SP, Brazil. \\ Associate Editor: Angelina Zanesco (D), ${ }^{1}$ Universidade Metropolitana de Santos, Faculdade de \\ Medicina, Santos, SP, Brazil; Universidade Estadual Paulista "Júlio de Mesquita Filho", \\ Departamento de Educação Física, Instituto de Biociências, Rio Claro, SP, Brazil. E-mail: \\ angelina.zanesco@unesp.br.
}

\begin{abstract}
Aim: This quasi-experimental study, analyzes if periodized combined training's influence on plasma cytokine levels, muscle strength, and cardiorespiratory fitness in physically active women with overweight and/or obesity over 50 years. Methods: The sample was composed of 33 physically active women who were randomly allocated to three groups: Non-Periodized (NP) ( $=11)$; Undulating Periodization (UP) $(n=9)$; and Flexible Undulating Periodization (FUP) $(\mathrm{n}=13)$. This study was conducted for 17 weeks, which included adaptation (weeks 1-3), baseline testing (week 4), training program (weeks 5-16), and post-evaluation (week 17). The training frequency comprised of three weekly sessions, with $30 \mathrm{~min}$ of aerobic exercise and $45 \mathrm{~min}$ of strength exercises. The data were analyzed using descriptive statistics and analysis of variance. The level of significance was $p \leq 0.05$. Results: No differences were observed in weight parameters, plasma levels of cytokines, and biochemical parameters in any group, before and after the completion of the training period. Participants demonstrated increase in strength of the upper limbs in the UP $(p=0.032)$ and FUP $(p=0.021)$ groups, an increase in aerobic fitness in the NP $(p=0.041)$ and UP $(p=0.005)$ groups, and an increase in the amount of time of vigorous activity per week in the NP group $(p=0.019)$. Conclusion: This study shows that periodized combined training in physically active women with overweight and/or obesity over 50 years proved to be beneficial for all groups; although, the UP group exhibited better performance.
\end{abstract}

Keywords: cytokines, resistance training, cardiorespiratory fitness, circuit-based exercise, aging.

\section{Introduction}

Obesity, a chronic non-communicable disease, contributes to the development of metabolic syndrome and adverse medical conditions, including type 2 diabetes and cardiovascular problems ${ }^{1}$.

In the absence of overt disease, obesity is often associated with increased levels of inflammatory markers and adipokines, as well as low-grade chronic systemic inflammation that seems to increase with age ${ }^{2}$. However, regular exercise has demonstrated a protective effect against diseases associated with this type of inflammation as well as in symptoms related to metabolic syndrome and sarcopenia $^{3,4}$. Therefore, it is used as an adjuvant in the prevention and treatment of various diseases ${ }^{5}$.

The anti-inflammatory effect of exercise is mediated by interleukin 6 (IL-6), which is generated and released when muscle fibers contract. The increase in plasma levels of IL-6 indirectly stimulates the synthesis of anti-inflammatory cytokines like interleukin 10 (IL-10), which inhibits the production of proinflammatory cytokines like tumor necrosis factor-alpha $(\mathrm{TNF}-\alpha)^{3,6}$.

Studies point at the fact that women above the age of 50 are more likely to be overweight or obese ${ }^{2}$ as a result of the natural aging process. Nevertheless, older persons are increasingly participating in cardiorespiratory fitness and strength exercises to keep themselves fit $^{7}$, which can help prevent or control sarcopenia, body weight gain, and lowgrade chronic systemic inflammation ${ }^{3,4}$. Additionally, when aerobic and strength exercises are performed in the same training session, they can be classified as combined training, that proves beneficial to one's health ${ }^{8}$.

Additionally, periodization, i.e., general and comprehensive planning of available training time based on the desired outcome, can be feasible and beneficial to inactive adults and older persons seeking to improve their 
health ${ }^{9}$. Therefore, this study observed the periodized combined training in three training groups: Non-Periodized (NP), Undulating Periodization (UP), and Flexible Undulating Periodization (FUP). In a NP model, there was no systematic variation in volume and intensity ${ }^{9}$. UP was characterized by more frequent changes in intensity and volume, which could be done weekly or daily, and these changes were visible in a period of 7-10 days ${ }^{9}$. FUP, similar to UP in terms of volume and intensity distribution, allowed the intensity and daily volume of the training load to be adjusted based on the individual's general state .

A combination of aerobic and strength training, for older persons, has more benefits in terms of increased aerobic fitness, strength, agility, and balance, and has been proven essential for healthy aging ${ }^{10}$. Several studies of periodized training in women, however, included interventions that focused solely on strength training and were targeted at the young adult population ${ }^{11}$. Studies conducted on women aged over 50 years -primarily for sedentary women-have mainly related periodization to strength training without correlating it with combined training, i.e., aerobic and strength training during the same session ${ }^{12}$.

Therefore, based on the benefits that lifestyle changes can bring to the health of the older, impact of exercise on people's health, and to emphasize the reduction of the inflammatory state along with the improvement of physical fitness components, there is great interest in analyzing whether periodized combined training influences plasma levels of proinflammatory cytokines, muscle strength, and aerobic fitness in this population. However, few studies have evaluated the effect of different periodization in combined training in physically active overweight women over 50 years analyzing whether this kind of training influences plasma cytokine levels, muscle strength, and cardiorespiratory fitness, since most studies are related only to combined training (but not periodized) or periodized training only related to strength exercises, and were carried out with men ${ }^{13,14}$; even when the target population was women, young adults and sedentary women were examined, leaving out those that were overweight and/or obese ${ }^{15}$.

The FUP model allowed participants to choose the order of sessions considering their daily physiological and psychological conditions, which gave them partial autonomy over the training program and might increase their motivation and effort input ${ }^{16}$. Thus, we hypothesized that the FUP model would achieve the best results related to the UP and NP models.

Thus, this study aimed to analyze whether periodized combined training influences plasma cytokine levels, muscle strength, and cardiorespiratory fitness in physically active women with overweight and/or obesity over 50 years.

\section{Methods}

This study was approved by the Research Ethics Committee, under the Brazilian National Health Council Resolution 466/2012, which regulates research involving human participants (CAAE: 56988216.2.0000.5393). All participants signed an informed consent form.

\section{Participants}

This was a quasi-experimental study, involving physically active overweight and/or obese women (body mass index (BMI) $\geq 25 \mathrm{~kg} / \mathrm{m}^{2}$ ), between the age of $50-70$ years. Data from a pilot study ( $\mathrm{n}=10$ for each group) helped in determining the variability to calculate the size of the definitive sample, considering the power of the test to be $80 \%$ with a significance level of $5 \%(\mathrm{p}<0.05)$.

The inclusion criteria comprised of active overweight and/or obese women with a BMI $\geq 25 \mathrm{~kg} / \mathrm{m}^{2}$, aged between 50-70 years, who exercised regularly (a combination of strength and aerobic training) for at least six months for a minimum of 150 min per week, and were able to train as per the medical certification they provided. The study demanded them be available to participate in the School of Physical Education and Sport of Ribeirão Preto, University of São Paulo's "Physical Education Program for the Elderly". Exclusion criteria included the presence of any medical, psychological, or musculoskeletal conditions that could impair their performance in strength training and aerobic exercises; those suffering from 3 or more non-communicable diseases, concurrently; consecutive falls; and being unable to achieve a minimum frequency of $\geq 70 \%$ participation in the training program ${ }^{17}$.

The initial sample consisted of 55 women who attended the inclusion criteria. After baseline testing, participants were randomly assigned, by sortition, into three groups: $\mathrm{NP}(\mathrm{n}=18,32,7 \%)$, UP $(\mathrm{n}=18,32,7 \%)$, and FUP $(\mathrm{n}=19,34,6 \%)$ participants.

Of the 55 participants in the training groups, 22 were excluded for the following reasons: dropped out due to pre-existing pain $(\mathrm{n}=5)$, did not complete the $\geq 70 \%$ frequency in training $(\mathrm{n}=12)$, and did not have blood samples taken at baseline or in the post-evaluation $(n=5)$; thus, the final sample of this study consisted of 33 women who continued to be assigned into their previously defined groups: NP $(\mathrm{n}=11,33.3 \%)$, UP $(\mathrm{n}=9,27.3 \%)$, and FUP $(\mathrm{n}=13,39.4 \%)$. The average age of the three groups was $60.8 \pm 6.6$ years.

\section{Training program}

The training program was conducted for 17 weeks, this study was divided into 4 phases: adaptation, pre-evaluation, intervention, and post-evaluation (Figure 1).

During the first phase, participants went through a three-week adaptation period so they could get used to the biomechanics of the exercises that would be performed. In 


\begin{tabular}{|c|c|c|c|}
\hline Adaptation & $\begin{array}{c}\text { Pre-evaluation } \\
\text { (Baseline testing) } \\
\text { Weeks 1-3 }\end{array}$ & $\begin{array}{c}\text { Intervention: NP, UP, } \\
\text { and FUP } \\
\text { Weeks 5-16 }\end{array}$ & \begin{tabular}{|c|} 
Post-evaluation \\
Week 17
\end{tabular} \\
\hline
\end{tabular}

Figure 1 - Experimental design: NP: Non-Periodized group; UP: Undulating Periodization Group; FUP: Flexible Undulating Periodization group.

this phase, aerobic and strength exercises that were similar to the training program were conducted. During the second phase, baseline testing, clinical tests, and analyzes were performed (fourth week), the participants were randomly assigned, by sortition, into three groups (NP, UP, and FUP). The intervention began during the third phase of the training program, which lasted 12 weeks (weeks 516). Post-evaluation began in the final phase of the program and participants were reassessed for clinical tests and analysis (week17).

The training program (intervention program) consisted of 2 sets of 8 exercises plus $30 \mathrm{~min}$ of aerobic exercise. Training sessions lasted for $1 \mathrm{hr} 15 \mathrm{~min}$, with $30 \mathrm{~min}$ of aerobic exercise on a treadmill or ergometer cycling and 45 min of strength exercises. Three weekly sessions were scheduled and always occurred at the same time. Participants were grouped in such a way that, if in the first training session they started with aerobic exercise, in the second, they would start with strength exercises, vice versa. Alternating the order in which exercises were performed made it possible to avoid bias in terms of strength gain.

For all the groups, strength training intensity was gradually increased when the participants were able to smoothly perform more repetitions than the target zone (10-12 repetition maximum, RM, for example, using the maximum load to perform 10-12 repetitions until concentric failure).

In strength training, in each training session, participants started on a different weight training machine from the eight previously selected ones, ensuring that there was no preference between the muscle groups, i.e., the sequence was performed from the smallest to the largest muscle group, vice versa, thus avoiding a possible bias in the strength gain, as the order of exercises can interfere in the increase in muscle strength $^{18}$. Weight training machines were labeled 1 to 8 : 1 -inclined bench press with free bar; 2-leg curl; 3-leg extension; 4-horizontal leg press; 5-rower with open grip; 6-lat pull-down; 7-triceps on the cross over using the triangle device 8-biceps curl with dumbbells.

In aerobic training, at the end of $30 \mathrm{~min}$ of exercise, the rating of perceived exertion (RPE) was used to verify that the exercise intensity was in line with the performed training. A frequency meter was used to check the reliability of the RPE data. Heart rate reserve (HRR) was eligible as the first criterion, and RPE was considered mainly for subjects who used medicines that modulate heart rate. HRR was calculated as the maximum heart rate from the maximum cardiopulmonary exercise test and resting heart rate.

\section{Training groups}

UP: In the strength training, the first session consisted of 2 sets of 5 to $7 \mathrm{RM}$, the second session, 2 sets of 10 to $12 \mathrm{RM}$, and the third session, 2 sets of 15 to $17 \mathrm{RM}$, providing different stimuli to the muscles so that strength training, hypertrophy, and muscle strength would improve ${ }^{9}$. In the aerobic training, intensities were 15 to 16 (hard) in the RPE and/or 70\% of the HHR on the first day, 13 to 14 (somewhat hard) and/or $60 \%$ of the HHR on the second day, and 11 to 12 (light) and/or $50 \%$ of the HHR on the third day.

FUP: Is similar to UP following the same protocol of 3 sessions but considering the individual's daily physiological variation and mental conditions, allowing them to have some autonomy by choosing the order of their training ${ }^{9,16}$, i.e., as the order of sessions was not fixed, participants would choose the order of the three sessions they would perform. If in the first session of strength training, they decided to do 15 to $17 \mathrm{RM}$, in the next they would have the option of 5 to $7 \mathrm{RM}$ or 10 to $12 \mathrm{RM}$ (on the last training day of the week there was no option to choose). Aerobic training followed the same pattern: if in the first session, participants decided to perform the training with an intensity of 11 to 12 (light) in the RPE and/or $50 \%$ of the HHR, in the next, they would have as an option 13 to 14 (somewhat hard) and/or $60 \%$ of the HHR, and 15 to 16 (hard) and/or $70 \%$ of the HHR. In the third session, there was no option to choose from.

NP: There were no variations in volume and intensity, maintaining a pattern of 10 to $12 \mathrm{RM}$ in strength and aerobic training intensity of 13 to 14 (somewhat hard) in RPE (and/or $60 \%$ of the HHR).

It was decided not to use a control group because the participants were already active and had been practicing regular exercises (strength and aerobic exercises) for at least six months for a minimum of 150 min per week. As already observed in the literature, the practice of regular exercise provides health benefits ${ }^{5,7,8,10}$. Thus, the NP group, in which there were no variations in volume and intensity, was used for comparison.

\section{Variables}

Sociodemographic and clinical data

To analyze sociodemographic variables and the history of diseases, a questionnaire prepared by the research group was used. Regarding sociodemographic variables, the following were considered: name, gender, age, years 
of study and schooling level, and concerning the history of diseases, metabolic-related diseases such as hypertension, diabetes mellitus, and dyslipidemia self-reported by the participants were considered, as well as the medications used for these chronic non-communicable diseases.

Body weight analysis

The weight variables analyzed in the present study were body weight, body mass index (BMI), and waist circumference (WC).

Bodyweight: it was expressed in kilograms $(\mathrm{kg})$ and measured using a Camry digital scale.

BMI: it was used the international classification of overweight and obesity for adults according to the BMI (European descendants) - overweight: $25-29.9 \mathrm{~kg} / \mathrm{m}^{2}{ }^{19}$.

WC: It was evaluated using a tape measure for the circumference at the midpoint between the last costal arch and the iliac crest, and the obtained value was recorded in centimeters. The classification used for $\mathrm{WC}$ was one that presents a risk for the development of metabolic diseases in participants, with a value of $\geq 80 \mathrm{~cm}$ for women ${ }^{19,20}$.

Blood analysis

For the analysis of biochemical parameters were considered: fasting glucose, total cholesterol (TC), highdensity lipoprotein (HDL) cholesterol, low-density lipoprotein (LDL) cholesterol, and triglycerides (TG). To evaluate the inflammatory response of exercise were analyzed TNF- $\alpha$, considered pro-inflammatory, and IL-6, considered pro- and anti-inflammatory. Cytokines were analyzed using the Enzyme-Linked Immunosorbent Assay method - ELISA, using commercial kits. The IL-6 cytokine was analyzed using the R\&D Systems kit (Minneapolis, MN, United States), and TNF- $\alpha$ was analyzed with the Biolegend kit (San Diego, CA, United States), as specified by the manufacturers.

Physical tests

Muscle strength: to assess maximal muscle strength, the estimate of the maximum dynamic load was utilized (prediction of 1RM) using the Brzycki equation ${ }^{21}$. The initial and final parameters of the participants' strength were observed in the inclined bench press to measure strength in the upper limbs and in the horizontal leg press to measure strength in the lower limbs. Participants were instructed not to perform any other type of exercise before the test application. Before starting the test, a general warm-up was performed on an ergometer cycling for $5 \mathrm{~min}$. At the beginning of the test, 15 repetitions were performed with a light intensity on the weight training machine; then, a submaximal weight was selected according to the load used in the adaptation phase, and participants were instructed to properly perform the most repetitions as possible. When participants managed to perform at least 2 and no more than 10 repetitions, the test stopped, and when they could perform more than 10 repetitions, after 3 min rest, the test was repeated with a higher load. Aerobic fitness: aerobic fitness was assessed using the 6-min walk test ${ }^{22}$.

Physical activity levels

To assess habitual physical activity levels and min of physical activity performed per week it was used the International Physical Activity Questionnaire (IPAQ)Short Form ${ }^{23}$. Questions in this questionnaire are related to the time that the individual spends doing physical activity in the last week, and it classifies the individual as inactive (sedentary), irregularly active (insufficiently active), active, and very active (highly active) ${ }^{23}$.

The IPAQ-Short Form includes the four components of physical activity: physical activity at work, physical activity as a means of transport, physical activity at home (work, housework, and family care,) and leisure-time physical activity (sport and recreation), classified as walking, moderate and vigorous, according to the participants. The classification of physical activity level was obtained by adding the frequency and duration of all activities ${ }^{23}$.

\section{Data collection}

The following data collection procedures were assessed at the baseline testing (pre-evaluation), and after the training program was completed (post-evaluation): blood samples for analysis of biochemical parameters and cytokine levels; measurements of body weight, BMI, and WC; along with the estimate of maximum dynamic load test for upper and lower limbs; the 6-min walk test and IPAQ-Short Form.

\section{Statistical analysis}

Data were analyzed using descriptive statistics and analysis of variance. ANOVA is based on partitioning the total variance of a given response (dependent variable) into two parts: the first due to the regression model (in this case, between groups), and the second due to the residuals (errors) (within groups). The greater the gap between the group means in proportion to the first, the more evidence of the difference between the group means. This model assumes that its residuals have a normal distribution with a mean of 0 and constant variance. However, when this assumption was not met, a transformation was applied to the response variable. This procedure was performed using SAS® 9.4 software, using PROC GLM. For comparisons between groups 2 to 2 separated by time, orthogonal contrasts based on the $t$ distribution were used. When comparing groups and times, a $95 \%$ confidence interval $(95 \% \mathrm{CI})$ was considered. In all analyses, a significance level of $5 \%(\mathrm{p}<0.05)$ was used. To verify the association between qualitative variables, data were submitted to Fisher's exact test. 


\section{Results}

Concerning sociodemographic variables, participants were aged between 50 and 70 years and the mean age between the three groups was $60.8 \pm 6.6$ years. Regarding years of study, the mean between the three groups was $12.6 \pm 4.9$ years. As for the schooling level, it was found that $2(6.0 \%)$ participants had an incomplete elementary school, $3(9.1 \%)$ had completed elementary school, $2(6.1 \%)$ had not completed high school, 12 (36.4\%) had complete high school, 12 (36.4\%) complete higher education and $2(6.0 \%)$ postgraduate studies.

The presence of metabolic diseases was observed in 26 participants (78.8\%), and 57.6\% (19 participants) had hypertension, $45.5 \%$ (15 participants) dyslipidemia, and $15.2 \%$ (5 participants) diabetes mellitus, noting that they could present up to 3 diseases concurrently. 26 participants (78.8\%) used medication: $57.6 \%$ (19 participants) used antihypertensive drugs; $27.3 \%$ (9 participants) used statins, fibrates for triglycerides, and omega 3 ; and 9.1\% (3 participants) used antihyperglycemic agents. Regarding the use of cigarettes, it was observed that only one participant was a smoker (3.0\%).

Regarding weight variables, when groups were compared before and after the training period (time 1 or T1 vs. time 2 or T2, respectively), no significant differences were observed for body weight, BMI, or WC (Table 1). When the three groups were compared before the training period (time 1) and after the training period (time 2), also no significant differences were observed for the variables body weight, BMI, and WC.

In biochemical variables, when groups were compared before and after training (time 1 vs. time 2), there was no significant difference in the values of fasting glucose, TC, HDL, LDL, and TG (Table 2).

When the three groups were compared before the training period (time 1), a significant difference was observed only in the fasting glucose variable of the UP group compared to the NP (NP vs. UP $=0.009$; NP vs. FUP: $p=0.259$; UP vs. FUP: $p=0.084$ ). However, when the three groups were compared after the training period (time 2), no significant differences were observed in the biochemical variables.

Regarding cytokine levels, when groups were compared before and after training (time 1 vs. time 2), no significant difference was observed in the levels of IL- 6 and TNF- $\alpha$ (Table 3).

When comparing the three groups before the training period (time 1), there were also no significant differences for IL-6 (NP vs. UP: $p=0.611$;P vs. FUP: $p=0.155$; UP vs. FUP: $p=0.450)$ nor the TNF- $\alpha$ (NP vs. UP: $p=0.842$; NP vs. FUP: $p=0.244$; UP vs. FUP: $p=0.611$ ). When comparing the three groups after the training period (time 2 ), again, there were no significant differences for IL-6 (NP vs. UP: $p=0.957$; NP vs. FUP: $p=0.749$; UP vs. FUP: $\mathrm{p}=1.000$ ); nor the TNF- $\alpha$ (NP vs. UP: $\mathrm{p}=0.425$; NP vs. FUP: $p=0.843$; UP vs. FUP: $p=0.594)$.

Regarding muscle strength, when groups were compared before and after training (time 1 vs. time 2), the estimate of the maximum dynamic load conducted in the inclined bench press showed a significant increase in strength of the upper limbs in the UP $(p=0.032)$ and FUP $(p=0.021)$ groups. However, the test performed on the leg press showed no significant difference in the strength of the lower limbs.

When the three groups were compared before the training period (time 1), the estimate of the maximum dynamic load performed on the inclined bench press presented no significant differences in upper limbs strength (NP vs. UP: $p=0.911$; NP vs. FUP: $p=0.701$; UP vs. FUP: $p=0.780$ ); nor in lower limbs strength performed on the leg press (NP vs. UP: $p=0.103$; NP vs. FUP: $\mathrm{p}=0.210$; UP vs. FUP: $\mathrm{p}=0.606$ ).

Table 1 - Distribution of the participants regarding weight variables, by physical training group and evaluation time.

\begin{tabular}{|c|c|c|c|c|c|c|}
\hline \multirow[t]{3}{*}{ Variables } & \multicolumn{2}{|c|}{ Nonperiodization (NP) } & \multicolumn{2}{|c|}{ Undulating periodization (UP) } & \multicolumn{2}{|c|}{ Flexible undulating periodization (FUP) } \\
\hline & \multicolumn{2}{|c|}{$\mathbf{N}=11$} & \multicolumn{2}{|c|}{$\mathbf{N}=\mathbf{0 9}$} & \multicolumn{2}{|c|}{$\mathbf{N}=\mathbf{1 3}$} \\
\hline & $\mathbf{T 1}$ & $\mathbf{T 2}$ & T1 & $\mathbf{T 2}$ & T1 & $\mathbf{T} 2$ \\
\hline & Mean (SD) & Mean (SD) & Mean (SD) & Mean (SD) & Mean (SD) & Mean (SD) \\
\hline Bodyweight & $73.8(5.8)$ & $73.0(5.2)$ & $74.8(7.8)$ & $75.7(8.6)$ & $75.8(4.1)$ & $76.0(4.4)$ \\
\hline p-value & \multicolumn{2}{|c|}{0.753} & \multicolumn{2}{|c|}{0.734} & \multicolumn{2}{|c|}{0.915} \\
\hline BMI & $28.6(2.1)$ & $28.3(2.1)$ & $28.7(2.3)$ & $29.0(2.5)$ & $29.8(2.7)$ & $30.0(2.9)$ \\
\hline p-value & \multicolumn{2}{|c|}{0.791} & \multicolumn{2}{|c|}{0.759} & \multicolumn{2}{|c|}{0.904} \\
\hline WC & $98.8(8.7)$ & $96.0(7.2)$ & $94.3(6.5)$ & $96.4(6.7)$ & $97.8(8.1)$ & $98.5(6.5)$ \\
\hline p-value & \multicolumn{2}{|c|}{0.360} & \multicolumn{2}{|c|}{0.538} & \multicolumn{2}{|c|}{0.803} \\
\hline
\end{tabular}

Legend: Bodyweight is expressed in $\mathrm{kg}$; $\mathrm{BMI}$ is expressed in $\mathrm{kg} / \mathrm{m}^{2}$; $\mathrm{WC}$ is expressed in $\mathrm{cm}$. 
Table 2 - Distribution of the participants regarding biochemical variables, by physical training group and evaluation time.

\begin{tabular}{|c|c|c|c|c|c|c|}
\hline \multirow[t]{3}{*}{ Variables } & \multirow{2}{*}{\multicolumn{2}{|c|}{$\begin{array}{c}\text { Nonperiodization (NP) } \\
\mathbf{N}=\mathbf{1 1}\end{array}$}} & \multirow{2}{*}{\multicolumn{2}{|c|}{$\begin{array}{c}\text { Undulating periodization (UP) } \\
\mathrm{N}=09\end{array}$}} & \multicolumn{2}{|c|}{ Flexible undulating periodization (FUP) } \\
\hline & & & & & & $\mathbf{N}=\mathbf{1 3}$ \\
\hline & T1 & $\mathbf{T 2}$ & T1 & T2 & T1 & T2 \\
\hline & Mean (SD) & Mean (SD) & Mean (SD) & Mean (SD) & Mean (SD) & Mean (SD) \\
\hline \multicolumn{7}{|l|}{ Biochemical variables } \\
\hline Fasting glucose (mg/dL) & $101.1(12.3)$ & $94.9(6.8)$ & $88.6(5.0)$ & $88.2(7.6)$ & $96.5(13.3)$ & $95.5(12.0)$ \\
\hline p-value & \multicolumn{2}{|c|}{0.149} & \multicolumn{2}{|c|}{0.933} & \multicolumn{2}{|r|}{0.809} \\
\hline $\mathrm{TC}(\mathrm{mg} / \mathrm{dL})$ & $205.4(33.7)$ & $201.2(39.4)$ & $192.8(34.6)$ & $189.0(35.8)$ & $189.4(35.7)$ & $183.7(38.5)$ \\
\hline p-value & \multicolumn{2}{|c|}{0.778} & \multicolumn{2}{|c|}{0.824} & \multicolumn{2}{|r|}{0.682} \\
\hline $\mathrm{HDL}(\mathrm{mg} / \mathrm{dL})$ & $52.7(10.6)$ & $49.5(12.1)$ & $51.6(5.9)$ & $51.4(9.2)$ & $47.2(12.7)$ & $44.9(9.9)$ \\
\hline p-value & \multicolumn{2}{|c|}{0.450} & \multicolumn{2}{|c|}{0.970} & \multicolumn{2}{|r|}{0.582} \\
\hline LDL (mg/dL) & $128.3(28.4)$ & $126.6(34.7)$ & $116.2(30.1)$ & $116.3(28.8)$ & $116.6(29.7)$ & $112.9(36.7)$ \\
\hline p-value & \multicolumn{2}{|c|}{0.897} & \multicolumn{2}{|c|}{0.995} & \multicolumn{2}{|r|}{0.764} \\
\hline $\mathrm{TG}(\mathrm{mg} / \mathrm{dL})$ & $121.9(41.8)$ & $125.7(36.3)$ & $124.5(45.4)$ & $6.2(41.1)$ & $128.4(66.7)$ & $129.2(59.4)$ \\
\hline p-value & \multicolumn{2}{|c|}{0.854} & \multicolumn{2}{|c|}{0.441} & \multicolumn{2}{|r|}{0.968} \\
\hline
\end{tabular}

Legend: TC: total cholesterol; HDL: high-density lipoprotein cholesterol; LDL: low-density lipoprotein cholesterol; TG: triglycerides.

Table 3 - Distribution of the participants regarding IL-6 and TNF- $\alpha$ levels, by physical training group and evaluation time.

\begin{tabular}{|c|c|c|c|c|c|c|}
\hline \multirow[t]{3}{*}{ Variables } & \multirow{2}{*}{\multicolumn{2}{|c|}{$\begin{array}{c}\text { Nonperiodization (NP) } \\
\mathbf{N}=\mathbf{1 1}\end{array}$}} & \multirow{2}{*}{\multicolumn{2}{|c|}{$\begin{array}{c}\text { Undulating periodization (UP) } \\
\mathrm{N}=09\end{array}$}} & \multicolumn{2}{|c|}{ Flexible undulating periodization (FUP) } \\
\hline & & & & & \multicolumn{2}{|c|}{$\mathbf{N}=\mathbf{1 3}$} \\
\hline & T1 & $\mathbf{T} 2$ & T1 & $\mathbf{T 2}$ & T1 & $\mathbf{T 2}$ \\
\hline & Mean (SD) & Mean (SD) & Mean (SD) & Mean (SD) & Mean (SD) & Mean (SD) \\
\hline IL-6 (pg/mL) & $3.5(3.2)$ & $4.6(4.9)$ & $4.6(4.6)$ & $4.4(4.7)$ & $3.8(3.3)$ & $3.3(1.9)$ \\
\hline p-value & \multicolumn{2}{|c|}{0.653} & \multicolumn{2}{|c|}{1.000} & \multicolumn{2}{|c|}{0.722} \\
\hline $\mathrm{TNF}-\alpha(\mathrm{pg} / \mathrm{mL})$ & $2.0(1.2)$ & $3.3(3.2)$ & $2.7(2.4)$ & $3.7(2.0)$ & $2.2(2.1)$ & $4.2(4.4)$ \\
\hline p-value & \multicolumn{2}{|c|}{0.469} & \multicolumn{2}{|c|}{0.258} & \multicolumn{2}{|c|}{0.211} \\
\hline
\end{tabular}

When comparing the three groups after the training period (time 2), the estimate of the maximum dynamic load performed on the inclined bench press, showed no significant differences in upper limbs strength (NP vs. UP: $p=0.354$; NP vs. FUP: $p=0.222$; UP vs. FUP: $p=0.818$ ). Nevertheless, a significant increase in lower limbs strength was observed in the leg press when the UP group was compared with the NP group, and when the FUP group was compared with the NP group (NP vs. UP: $p=0.016$; NP vs. FUP: $p=0.035$; UP vs. FUP: $p=0.609$ ).

Regarding aerobic fitness, when groups were compared before and after training (time 1 vs. time 2), an increase in cardiorespiratory fitness was observed, with a significant difference for the NP group $(\mathrm{p}=0.041)$ and the UP group $(p=0.005)$ (Table 4$)$. When comparing the three groups before the training period (time 1), no significant differences were observed (NP vs. UP: $p=0.108$; NP vs. FUP: $p=0.180$; UP vs. FUP: $p=0.681$ ). However, when comparing the three groups after the training period (time 2), a significant improvement in cardiorespiratory fitness was observed in the 6-min walk test of the UP group compared to the NP group (NP vs. UP: $p=0.009$; NP vs. FUP: $\mathrm{p}=0.404$; UP vs. FUP: $\mathrm{p}=0.050$ ).

According to the classification conducted with the IPAQ-Short Form by comparing data recorded before and after the training period (time 1 vs. time 2), there was a reduction in the number of participants categorized as active, and an increase in the number of participants classified as highly active in all three groups (Table 5). 
Table 4 - Evaluation of the participants' strength as measured by the maximum dynamic load with the inclined bench press and leg press and assessment of aerobic fitness as measured in the 6-min walk test, by physical training group and evaluation time.

\begin{tabular}{|c|c|c|c|c|c|c|}
\hline \multirow[t]{2}{*}{ Variables } & \multicolumn{2}{|c|}{ Nonperiodization (NP) } & \multicolumn{2}{|c|}{ Undulating periodization (UP) } & \multicolumn{2}{|c|}{ Flexible undulating periodization (POF) } \\
\hline & T1 & $\mathbf{T} 2$ & T1 & $\mathbf{T} 2$ & T1 & $\mathbf{T} 2$ \\
\hline \multirow[t]{3}{*}{ MDL: Bench press } & $\mathrm{N}=8$ & $\mathrm{~N}=8$ & $\mathrm{~N}=9$ & $\mathrm{~N}=7$ & $\mathrm{~N}=10$ & $\mathrm{~N}=9$ \\
\hline & Mean (SD) & Mean (SD) & Mean (SD) & Mean (SD) & Mean (SD) & Mean (SD) \\
\hline & $24.8(4.0)$ & $28.4(6.4)$ & $25.1(5.8)$ & $30.9(5.9)$ & $25.8(3.5)$ & $31.5(7.3)$ \\
\hline p-value & \multicolumn{2}{|c|}{0.180} & \multicolumn{2}{|c|}{0.032} & \multicolumn{2}{|c|}{0.021} \\
\hline \multirow[t]{3}{*}{ MDL: Leg press } & $\mathrm{N}=8$ & $\mathrm{~N}=8$ & $\mathrm{~N}=8$ & $\mathrm{~N}=8$ & $\mathrm{~N}=11$ & $\mathrm{~N}=11$ \\
\hline & Mean (SD) & Mean (SD) & Mean (SD) & Mean (SD) & Mean (SD) & Mean (SD) \\
\hline & $123.8(19.6)$ & $128.5(15.5)$ & $142.2(20.3)$ & $156.0(23.4)$ & $136.8(28.0)$ & $150.7(27.2)$ \\
\hline p-value & \multicolumn{2}{|c|}{0.673} & \multicolumn{2}{|c|}{0.215} & \multicolumn{2}{|c|}{0.146} \\
\hline \multirow[t]{3}{*}{ Aerobic fitness: 6-min walk test } & $\mathrm{N}=11$ & $\mathrm{~N}=11$ & $\mathrm{~N}=09$ & $\mathrm{~N}=09$ & $\mathrm{~N}=13$ & $\mathrm{~N}=13$ \\
\hline & Mean (SD) & Mean (SD) & Mean (SD) & Mean (SD) & Mean (SD) & Mean (SD) \\
\hline & $551.8(47.3)$ & $600.5(49.6)$ & $591.8(34.3)$ & $665.2(71.2)$ & $582.2(58.6)$ & $618.9(64.1)$ \\
\hline p-value & & 0.041 & & 0.005 & & 0.085 \\
\hline
\end{tabular}

Legend: MDL (maximum dynamic load) is expressed in kg; Aerobic fitness-6-min walk test is expressed in min.

Table 5 - Classification of the physical activity level of the participants as measured using the IPAQ-Short Form, by physical training group and evaluation time.

\begin{tabular}{|c|c|c|c|c|c|c|}
\hline \multirow[t]{3}{*}{ Variables } & \multirow{2}{*}{\multicolumn{2}{|c|}{$\begin{array}{c}\text { Nonperiodization (NP) } \\
\mathbf{N}=11\end{array}$}} & \multirow{2}{*}{\multicolumn{2}{|c|}{$\begin{array}{c}\text { Undulating periodization (UP) } \\
\mathrm{N}=09\end{array}$}} & \multicolumn{2}{|c|}{ Flexible undulating periodization (FUP) } \\
\hline & & & & & \multicolumn{2}{|c|}{$\mathbf{N}=\mathbf{1 3}$} \\
\hline & T1 & $\mathbf{T 2}$ & T1 & $\mathbf{T 2}$ & T1 & $\mathbf{T 2}$ \\
\hline & $\mathrm{N}(\%)$ & $\mathrm{N}(\%)$ & $\mathrm{N}(\%)$ & $\mathrm{N}(\%)$ & $\mathrm{N}(\%)$ & $\mathrm{N}(\%)$ \\
\hline \multicolumn{7}{|l|}{ Classification } \\
\hline Irregularly active & $0(0.0)$ & $0(0.0)$ & $0(0.0)$ & $0(0.0)$ & $1(7.7)$ & $0(0.0)$ \\
\hline Active & $8(72.7)$ & $3(27.3)$ & $7(77.8)$ & $6(66.7)$ & $10(76.9)$ & $7(53.8)$ \\
\hline Highly active & $3(27.3)$ & $8(72.7)$ & $2(22.2)$ & $3(33.3)$ & $2(15.4)$ & $6(46.2)$ \\
\hline
\end{tabular}

Legend: Fisher's exact test was applied to T1 for frequency, and the association test for the physical activity level variable: $p$-value $=0.9490 ;$ Fisher's exact test was applied to T2 for frequency, and the association test for the physical activity level variable: $\mathrm{p}$-value $=0.2297$.

Regarding min of physical activities performed per week, when groups were compared before and after the training period (time 1 vs. time 2), no significant differences were observed in min of walking and moderate physical activity performed per week. However, it was observed a significant increase in min of vigorous physical activity performed per week in the NP group $(\mathrm{p}=0.019)$ (Table 6).

When the three groups were compared before the training period (time 1), no significant differences were observed in min of walking, min of moderate physical activity, or min of vigorous physical activity performed per week. Nevertheless, when comparing the three groups after the training period (time 2), there was a significant improvement in min of vigorous physical activity performed per week in the NP group compared to the FUP group (NP vs. UP: $\mathrm{p}=0.658$; NP vs. FUP: $\mathrm{p}=0.044$; UP vs. FUP: $p=0.144)$.

\section{Discussion}

This study assessed the periodized combined training to analyze whether this kind of training influences plasma cytokine levels, muscle strength, and cardiorespiratory fitness in physically active women with overweight and/or obesity over 50 years. Results did not confirm our hypothesis that the FUP model would achieve superior results related to the UP model and NP model. When comparing results before and after the training program, we observed that both UP and FUP groups presented an increase in strength of the upper limbs, NP and UP groups showed an increase in aerobic fitness, and the 
Table 6 - Distribution of the participants according to the min of physical activities performed per week as evaluated using the IPAQ-Short Form, by physical training group, and evaluation time.

\begin{tabular}{|c|c|c|c|c|c|c|}
\hline \multirow[t]{3}{*}{ Variables } & \multicolumn{2}{|c|}{ Nonperiodization (NP) } & \multicolumn{2}{|c|}{ Undulating periodization (UP) } & \multicolumn{2}{|c|}{ Flexible undulating periodization (FUP) } \\
\hline & \multicolumn{2}{|c|}{$\mathbf{N}=\mathbf{1 1}$} & \multicolumn{2}{|c|}{$\mathbf{N}=09$} & \multicolumn{2}{|c|}{$\mathbf{N}=\mathbf{1 3}$} \\
\hline & T1 & T2 & T1 & $\mathbf{T 2}$ & T1 & T2 \\
\hline & Mean (SD) & Mean (SD) & Mean (SD) & Mean (SD) & Mean (SD) & Mean (SD) \\
\hline Walk & $281.8(250.0)$ & $301.8(238.7)$ & $277.8(202.0)$ & $455.6(501.0)$ & $397.7(779.0)$ & $348.5(298.1)$ \\
\hline p-value & \multicolumn{2}{|c|}{0.912} & \multicolumn{2}{|c|}{0.377} & \multicolumn{2}{|c|}{0.768} \\
\hline Moderate PA & $538.6(535.9)$ & $480.0(576.5)$ & $460.0(278.6)$ & $516.7(524.0)$ & $414.6(326.3)$ & $376.6(342.9)$ \\
\hline p-value & \multicolumn{2}{|c|}{0.745} & \multicolumn{2}{|c|}{0.776} & \multicolumn{2}{|c|}{0.818} \\
\hline Vigorous PA & $57.3(70.3)$ & 200.9 (194.9) & $82.2(75.6)$ & $172.9(236.1)$ & $86.2(158.7)$ & $82.9(79.7)$ \\
\hline $\mathrm{p}$-value & \multicolumn{2}{|c|}{0.019} & \multicolumn{2}{|c|}{0.175} & \multicolumn{2}{|c|}{0.953} \\
\hline
\end{tabular}

Legend: The results refer to the min spent performing physical activity per week; PA: physical activity.

NP group presented an increase in min of vigorous activity carried out weekly. In general, all groups benefited from the exercise training program, and the UP group exhibited better performance.

In this study, the 12-week periodized combined training program did not affect weight parameters when groups were compared before and after training (time $1 \mathrm{vs.}$ time 2). Although all participants have reached the minimum frequency requirement of $70 \%$, the absence of a reduction in body weight, $\mathrm{BMI}$, and WC can be related to the maintenance of inadequate dietary habits, since it was not conducted nutritional follow-up for participants. Nutritional follow-up was not performed in this study, however, it was expected that the participants were aware and had maintained healthy eating habits on their own, since they were starting a training program in which body measurements, blood tests, and the inflammatory response would be assessed, in addition to physical tests related to strength and cardiorespiratory fitness.

In obese people age 65 and above, a diet or a diet combined with exercises resulted in a loss of body weight of about $10 \%$, however, no weight loss was observed in the group that did only exercises ${ }^{24}$, corroborating the findings of this study. Additionally, a study that evaluated, among other parameters, the effect of combined training on body weight in women age 35 with metabolic syndrome, also did not observe a reduction in this variable, even though the participants were younger ${ }^{15}$.

Although the participants in this study did not show changes in weight variables, it was observed in all groups that they evolved from active to highly active, according to the classification of the IPAQ-Short Form, a change that can be considered beneficial to health. According to the recommendation of the American College of Sports Medicine/American Heart Association, to promote and maintain adults' and older people's health, training must emphasize $150 \mathrm{~min} /$ week of aerobic exercise at moderate intensity for at least $30 \mathrm{~min}$, five times a week, or $60-75 \mathrm{~min} /$ week of high-intensity aerobic exercise for at least $20 \mathrm{~min}$, three times a week. Additionally, muscle strengthening exercises should also be performed at least twice a week to maintain or increase strength $^{25,26}$. The findings of the present study corroborate these recommendations.

Regarding biochemical variables, when groups were compared before and after the training program (time $1 \mathrm{vs.}$ time 2), no significant difference was observed in the values of fasting glucose, TC, HDL, LDL, and triglycerides. It is noted that despite the difference observed between the NP and UP groups at time 1, these values were within the normal parameters ${ }^{27}$. In agreement with the present data, one study did not observe statistical differences in biochemical parameters after 12 weeks of combined training ${ }^{28}$, and another study, after 21 weeks of combined training compared to aerobic training or strength training alone, neither observed any changes in blood lipids, blood pressure or serum glucose and insulin between groups ${ }^{29}$.

Concerning cytokine concentrations, no significant differences were observed in IL- 6 and TNF- $\alpha$ levels when groups were compared before and after the training period (time 1 vs. time 2), and neither when the three groups were compared to each other after the training period (time 2). The fact that participants were already physically active before the training period may have influenced the outcome, as the anti-inflammatory effect of the exercise might have already been reached, and there is a possibility that different results would have been achieved if the study had evaluated sedentary participants ${ }^{13}$. However, even though no positive results were observed, the participants probably benefited from the training, as exercise exerts an anti-inflammatory effect on the body. 
The effect of exercise on inflammatory response is well established in the literature ${ }^{3,4,30}$. This effect can be attributed to an anti-inflammatory response that is partially mediated by muscle-derived IL- 6 . The IL-6 physiological concentrations stimulate an increase in the circulating levels of anti-inflammatory cytokines, such as IL-10, and inhibit the production of the proinflammatory cytokine TNF- $\alpha^{3}$. Additionally, IL- 6 stimulates lipolysis in adipose tissue and fat oxidation ${ }^{3}$ and increases hepatic glucose production during exercise (gluconeogenesis) ${ }^{31}$. There is strong evidence that an increase in acute IL- 6 circulating levels improves fat oxidation, glucose absorption triggered by insulin, and has anti-inflammatory effects ${ }^{6}$. The antiinflammatory effects of exercise may protect against insulin resistance induced by TNF- $\alpha$, as it is suggested that regular exercise induces the suppression of this cytokine ${ }^{3}$.

Corroborating the findings of the current study regarding cytokine concentrations, a 10-week study of combined training conducted in women with metabolic syndrome did not report significant differences in IL-6 levels after the training program ${ }^{15}$. In addition, a 16-week study conducted with overweight men showed that moderate to high-intensity strength training and combined training did not result in a reduction in TNF- $\alpha$ and IL-6 concentrations ${ }^{14}$.

In this study, regarding muscle strength, it was observed that UP and FUP groups had an increase in upper limbs strength when compared before and after the training period (time 1 vs. time 2). And when the three groups were compared to each other at time 2, the UP and FUP groups showed a greater increase in lower limbs than the NP group. Alternatively, there was no significant increase in muscle strength of the lower limbs in the maximum dynamic load test evaluated in the leg press, when groups were compared before and after the training period (time 1 vs. time 2). However, it was probably because the lower limb muscles are used more daily, and already have a certain degree of trainability compared to the muscles of the upper limb. Nevertheless, regarding muscle strength, it can be said that the UP and FUP groups benefited more with the type of training conducted, as they had increased strength in the upper limbs when groups were compared before and after the training period and increased strength in the lower limbs when the three groups were compared at time 2. The NP group did not show an increase in muscle strength, neither in the comparison before and after the training period nor in the comparison between the groups.

Combined training has been shown to enhance aerobic fitness and strength in older adults ${ }^{8,32,33}$, and training periodization is a feasible and effective option for inactive adults and older persons aiming to improve their health conditions $^{9,12}$, as well as those in various stages of physical fitness and even those in rehabilitation ${ }^{34}$.

Consequently, in terms of aerobic fitness, the results of this study revealed that the NP and UP groups presented increased cardiorespiratory fitness when compared before and after the training period (time 1 vs. time 2 ). In addition, when the three groups were compared to each other at time 2, the UP group showed a significant improvement in cardiorespiratory fitness when compared to the NP group. Thus, the NP and UP groups benefited from their training program, as they had an increase in cardiorespiratory fitness when groups were compared before and after the training period (time 1 vs. time 2), and the UP group also showed an increase in cardiorespiratory fitness when the three groups were compared at time 2. Corroborating the findings of this study, an investigation observed that 21-week of aerobic training, strength training, or combined training led to specific improvements already expected in physical fitness (neuromuscular and cardiorespiratory) in healthy middle-aged and older women $^{29}$.

Therefore, it is important to mention that some studies have shown that combined training does not prejudice the development of aerobic fitness and can even enhance $\mathrm{it}^{8,35}$. However, other studies have suggested that this type of training seems to impair strength gains and muscle hypertrophy when compared exclusively with strength training ${ }^{36,37}$.

In this sense, a study that compared the effects of 16 weeks of strength training and combined training on muscle strength in middle-aged postmenopausal women, not physically active, observed an increase in maximum strength of the upper and lower limbs of both training groups, with no difference between them. There was an increase in $\mathrm{VO}_{2}$ peak only for the combined training. It was concluded that the combined training, conducted under the minimum aerobic training recommendations of the American College of Sports Medicine, did not influence maximum strength in postmenopausal middle-aged women $^{35}$. These results corroborate with the findings of the present study in terms of strength and aerobic fitness, remembering that in this study, women are physically active, and the order of exercises was inverted at each training session so that there was no influence on the results regarding the strength or aerobic fitness.

Periodization in physical training is usually evaluated in athletes, however, studies show that the prescription of periodized training can be feasible and beneficial for inactive adults as well as older adults, aiming at improving health conditions ${ }^{9,12}$. Therefore, this study assessed not only periodized training but periodized combined training to analyze whether this kind of training influences plasma cytokine levels, muscle strength, and cardiorespiratory fitness in physically active women with overweight and/or obesity over 50 years.

It is important to note that in this study, the positive results observed in some groups in both muscle strength and aerobic fitness indicate that health-related benefits were referring to the 12 -week periodized combined train- 
ing. This training program showed participants that they can work with load variations in strength training and carry out an aerobic activity at a greater intensity than they were accustomed to; moreover, improvements can be an incentive for them to continue to carry out exercises after the end of the program training.

However, one of the limitations of this study was the small sample size which may have negatively influenced the results. Nevertheless, more robust effects could not have been observed because the participants were already physically active before the beginning of the training program, and have gone through an adaptation period to familiarize themselves with the exercises that would be conducted during the training program, before the first assessment. Probably different results would have been observed if the participants were sedentary. Another point that can be considered a limitation is that we did not analyze hormone replacement.

It is worth noting that the differences between the types of training conducted, i.e., the total duration of the training program, the number of sets per week, the volume of strength training, the intensity of aerobic training, and even in the case of having conducted a periodized training, the kind of periodization that was applied, made it impossible for a comparison to other studies. It is suggested that further studies on periodized combined training in physically active overweight and/or obese women over 50 years; be conducted with larger populations and with nutritional monitoring.

\section{Conclusion}

Based on the findings of this study, although no significant differences were found in weight, biochemical and inflammatory variables in any of the groups when they were compared before and after training (time 1 vs. time 2 ), it was observed that the UP and FUP groups showed an increase in upper-limb strength, and the NP and UP groups showed an increase in aerobic fitness, while the NP group showed an increase in the min of vigorous activity performed per week. Therefore, it was concluded that the periodized combined training did not influence plasma cytokine levels on physically active women with overweight and/or obesity over 50 years, but was efficient in improving upper limbs strength of the UP and FUP groups, besides improving cardiorespiratory fitness of the NP and UP groups and increasing the min of vigorous activity conducted per week by the NP group when the groups were compared before and after the training program. It can be said that periodized combined training in physically active women with overweight and/or obesity proved to be beneficial for all groups and that the UP group benefited more in terms of the type of training conducted, as it showed an increase in upper limbs strength and aerobic fitness in the comparison before and after the training program.

\section{Acknowledgments}

Fundação de Amparo à Pesquisa do Estado de São Paulo for the financial support.

\section{References}

1. Blüher M. Obesity: global epidemiology and pathogenesis. Nat Rev Endocrinol. 2019;15(5):288-98. doi.

2. Pfeilschifter J, Koditz R, Pfohl M, Schatz H. Changes in proinflammatory cytokine activity after menopause. Endocr Rev. 2002;23(1):90-119. doi.

3. Petersen AMW, Pedersen BK. The anti-inflammatory effect of exercise. J Appl Physiol. 2005;98:1154-62. doi.

4. Petersen AMW, Pedersen BK. The role of IL-6 in mediating the anti-inflammatory effects of exercise. J Physiol Pharmacol. 2006;57(Suppl 10):43-51.

5. Pedersen BK, Saltin B. Exercise as medicine-evidence for prescribing exercise as therapy in 26 different chronic diseases. Scand J Med Sci Sports. 2015;25(Suppl 3):1-72. doi.

6. Pedersen BK, Febbraio MA. Muscle as an endocrine organ: Focus on muscle-derived interleukin-6. Physiol Rev. 2008;88:1379-406. doi

7. Botero JP, Prado WL, Guerra RLF, Speretta GFF, Leite RD, Prestes J, et al. Does aerobic exercise intensity affect healthrelated parameters in overweight women? Clin Physiol Funct Imaging. 2014;34(2):138-42. doi.

8. Guedes JM, Bortoluzzi MG, Matte LP, Andrade CM, Zulpo $\mathrm{NC}$, Sebben V, et al. Efeitos do treinamento combinado sobre a força, resistência e potência aeróbica em idosas. Rev Bras Med Esporte. 2016;22(6):480-4. doi.

9. Strohacker K, Fazzino D, Breslin WL, Xu X. The use of periodization in exercise prescriptions for inactive adults: a systematic review. Prev Med Rep. 2015;2:385-96. doi

10. Nelson ME, Rejeski WJ, Blair SN, Duncan PW, Judge JO, King AC et al. Physical activity and Public Health in Older Adults: recommendation from the American College of Sports Medicine and the American Heart Association. Med Sci Sports Exerc. 2007;39:1435-45.

11. Bartolomei S, Stout JR, Fukuda DH, Hoffman JR, Merni F. Block vs. weekly undulating periodized resistance training programs in women. J Strength Cond Res. 2015;29 (10):2679-87. doi.

12. Botero JP, Shiguemoto GE, Prestes J, Marin CT, Do Prado WL, Pontes C, et al. Effects of long-term periodized resistance training on body composition, leptin, resistin, and muscle strength in elderly post-menopausal women. J Sports Med Phys Fitness. 2013;53(3):289-94.

13. Brunelli DT, Chacon-Mikahil MPT, Gaspari AF, Lopes WA, Bonganha $\mathrm{V}$, Bonfante ILP, et al. Combined training reduces subclinical inflammation in middle-aged men. Med Sci Sports Exerc. 2015;47(10):2207-15. doi.

14. Libardi CA, Souza GV, Gaspari AF, Santos CF, Leite ST, Dias R, et al. Effects of concurrent training on interleukin-6, tumour necrosis factor-alpha and C-reactive protein in middle-aged men. J Sports Sci. 2011;29(14):1573-81. doi. 
15. Tibana RA, Nascimento DC, Sousa NMF, Souza VC, Durigan J, Vieira A, et al. Enhancing of women functional status with metabolic syndrome by cardioprotective and antiinflammatory effects of combined aerobic and resistance training. Plos One. 2014;9(11):e110160. doi

16. Colquhoun R J, Gai CM, Walters J, Brannon AR, Kilpatrick MW, D'Agostino DP, et al. Comparison of powerlifting performance in trained men using traditional and flexible daily undulating periodization. J Strength Cond Res. 2017;31 (2):283-91.

17. Johannsen NM, Swift DL, Lavie CJ, Earnest CP, Blair SN, Church, TS. Categorical analysis of the impact of aerobic and resistance exercise training, alone and in combination, on cardiorespiratory fitness levels in patients with Type 2 Diabetes: results from the HART-D study. Diabetes Care. 2013;36:3305-12.

18. Spineti J, Salles BF, Rhea MR, Lavigne D, Matta T, Miranda $\mathrm{F}$, et al. Influence of exercise order on maximum strength and muscle volume in nonlinear periodized resistance training. J Strength Cond Res. 2010;24(11):2962-9. doi.

19. World Health Organization. Obesity: preventing and managing the global epidemic. Report of a World Health Organization Consultation. WHO Technical Report Series 894. Geneva, WHO; 2000.

20. International Diabetes Federation. The IDF consensus worldwide definition of the Metabolic Syndrome. Brussels, International Diabetes Federation; 2006

21. Knutzen KM, Brilla LR, Caine D. Validity of 1RM prediction equations for older adults. J Strength Cond Res. 1999;13(3):242-6.

22. Rikli RE, Jones CJ. Teste de aptidão física para idosos. Barueri, Manole; 2008.

23. Matsudo S, Araujo T, Matsudo V, Andrade D, Andrade E, Oliveira LC, et al. Questionário Internacional de Atividade Física (I-PAQ): estudo de validade e reprodutibilidade no Brasil. Rev Bras Ativ Fís Saúde. 2001;6(2):5-18.

24. Villareal DT, Chode S, Parimi N, Sinacore DR, Hilton T, Armamento-Villareal R, et al. Weight loss, exercise, or both and physical function in obese older adults. $\mathrm{N}$ Engl $\mathrm{J}$ Med. 2011;364(13):1218-29. doi.

25. Garber CE, Blissmer B, Deschenes MR, Franklin BA, Lamonte MJ, Lee IM, et al. American College of Sports Medicine position stand. Quantity and quality of exercise for developing and maintaining cardiorespiratory, musculoskeletal, and neuromotor fitness in apparently healthy adults: guidance for prescribing exercise. Med Sci Sports Exerc. 2011;43(7):1334-59. doi.

26. Haskell WL, Lee I-M, Pate RR, Powell KE, Blair SN, Franklin BA, et al. Physical activity and public health: updated recommendation for adults from the American College of Sports Medicine and the American Heart Association. Med Sci Sports Exerc. 2007;39(8):1423-34. doi.

27. Sociedade Brasileira de Diabetes. Posicionamento Oficial SBD no 07/2019 - Abordagem da pessoa idosa com diabetes 2019/2020. São Paulo, Sociedade Brasileira de Diabetes; 2019 .
28. Campos ALP, Del Ponte LS, Cavalli AS, Afonso MR, Schild JFG, Reichert FF. Effects of concurrent training on health aspects of elderly women. Rev Bras Cineantropom Desempenho Hum. 2013;15(4):437-47. doi.

29. Sillanpaa E, Laaksonen DE, Hakkinen A, Karavirta L, Jensen B, Kraemer WJ, et al. Body composition, fitness, and metabolic health during strength and endurance training and their combination in middle-aged and older women. Eur $\mathrm{J}$ Appl Physiol. 2009;106:285-96. doi.

30. Gleeson M, Bishop NC, Stensel DJ, Lindley MR, Mastana SS, Nimmo MA. The anti-inflammatory effects of exercise: mechanisms and implications for the prevention and treatment of disease. Nat Rev Immunol. 2011;11(9):607-15. doi.

31. Pedersen BK, Fischer CP. Beneficial health effects of exercise - the role of IL- 6 as a myokine. Trends Pharmacol Sci. 2007;28(4):152-6. doi

32. Wood RH, Reyes R, Welsch MA, Favaloro-Sabatier J, Sabatier M, Lee CM, et al. Concurrent cardiovascular and resistance training in healthy older adults. Med Sci Sports Exerc. 2001;33(10):1751-8. doi

33. Takeshima N, Rogers ME, Islam MM, Yamauchi T, Watanabe E, Okada A. Effect of concurrent aerobic and resistance circuit exercise training on fitness in older adults. Eur J Appl Physiol. 2004;93:173-82. doi

34. Hoover DL, VanWye WR, Judge LW. Periodization and physical therapy: bridging the gap between training and rehabilitation. Phys Ther Sport. 2016;18:1-20. doi.

35. Lixandrão $\mathrm{M}$, Bonganha $\mathrm{V}$, Conceição MS, Libardi CA, Berton RPB, Cavaglieri CR, et al. Efeito do treinamento concorrente sobre a força e hipertrofia muscular de mulheres na pós-menopausa. Rev Bras Ativ Fís Saúde. 2012;17 (4):247-51. doi

36. Bell GJ, Syrotuik D, Martin TP, Burnham R, Quinney HA. Effect of concurrent strength and endurance training on skeletal muscle properties and hormone concentrations in humans. Eur J Appl Physiol. 2000;81:418-27. doi

37. Hunter GR, McCarthy JP, Bamman MM. Effects of resistance training on older adults. Sports Med. 2004;34(5):32948.

\section{Corresponding author}

Angelita Maria Stabile. Universidade de São Paulo Escola de Enfermagem de Ribeirão Preto, Ribeirão Preto, SP,

Brazil.

E-mail: angelita@eerp.usp.br.

Manuscript received on July 15, 2021

Manuscript accepted on January 3, 2022

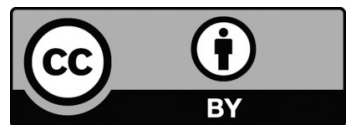

Motriz. The Journal of Physical Education. UNESP. Rio Claro, SP, Brazil - eISSN: 1980-6574 - under a license Creative Commons - Version 4.0 